\title{
Progesterone receptor membrane component-1 regulates hepcidin biosynthesis
}

\author{
Xiang Li, ${ }^{1,2}$ David K. Rhee, ${ }^{3}$ Rajeev Malhotra, ${ }^{3}$ Claire Mayeur, ${ }^{4}$ Liam A. Hurst, ${ }^{4}$ Emily Ager, ${ }^{4}$ Georgia Shelton, ${ }^{4}$ Yael Kramer, ${ }^{5}$ \\ David McCulloh, ${ }^{5}$ David Keefe, ${ }^{5}$ Kenneth D. Bloch, ${ }^{3,4}$ Donald B. Bloch, ${ }^{4,6}$ and Randall T. Peterson ${ }^{1,2}$ \\ 'Cardiovascular Research Center, Massachusetts General Hospital, Department of Systems Biology, Harvard Medical School, Boston, Massachusetts, USA. Broad Institute, Cambridge, Massachusetts, USA. \\ ${ }^{3}$ Cardiovascular Research Center and Division of Cardiology, Department of Medicine, and ${ }^{4}$ Anesthesia Center for Critical Care Research, Department of Anesthesia, Critical Care, and Pain Medicine, \\ Massachusetts General Hospital, Harvard Medical School, Boston, Massachusetts, USA. 'Department of Obstetrics and Gynecology, New York University Langone Medical Center, New York, New York, USA. \\ ${ }^{6}$ Center for Immunology and Inflammatory Diseases, Division of Rheumatology, Allergy, and Immunology, Department of Medicine, Massachusetts Ceneral Hospital, \\ Harvard Medical School, Boston, Massachusetts, USA.
}

\begin{abstract}
Iron homeostasis is tightly regulated by the membrane iron exporter ferroportin and its regulatory peptide hormone hepcidin. The hepcidin/ferroportin axis is considered a promising therapeutic target for the treatment of diseases of iron overload or deficiency. Here, we conducted a chemical screen in zebrafish to identify small molecules that decrease ferroportin protein levels. The chemical screen led to the identification of 3 steroid molecules, epitiostanol, progesterone, and mifepristone, which decrease ferroportin levels by increasing the biosynthesis of hepcidin. These hepcidin-inducing steroids (HISs) did not activate known hepcidin-inducing pathways, including the BMP and JAK/STAT3 pathways. Progesterone receptor membrane component-1 (PCRMC1) was required for HIS-dependent increases in hepcidin biosynthesis, as PCRMC1 depletion in cultured hepatoma cells and zebrafish blocked the ability of HISs to increase hepcidin mRNA levels. Neutralizing antibodies directed against PCRMC1 attenuated the ability of HISs to induce hepcidin gene expression. Inhibiting the kinases of the SRC family, which are downstream of PGRMC1, blocked the ability of HISs to increase hepcidin mRNA levels. Furthermore, HIS treatment increased hepcidin biosynthesis in mice and humans. Together, these data indicate that PCRMC1 regulates hepcidin gene expression through an evolutionarily conserved mechanism. These studies have identified drug candidates and potential therapeutic targets for the treatment of diseases of abnormal iron metabolism.
\end{abstract}

\section{Introduction}

Hepcidin, a small peptide hormone produced and secreted by hepatocytes, is an important regulator of iron metabolism in vertebrates $(1,2)$. Hepcidin binds to the only known iron exporter in vertebrates, ferroportin, and causes its internalization and ubiquitin-mediated (ubi-mediated) degradation (3). The interaction between hepcidin and ferroportin regulates iron absorption by duodenal enterocytes, the recycling of iron by macrophages, and iron release by hepatocytes $(1,2)$.

Hepcidin gene expression is induced by iron excess, lipopolysaccharide, inflammatory cytokines (e.g., IL-6), and BMPs and is decreased in response to hypoxia, iron deficiency, and acute blood loss $(1,4)$. Previously identified stimuli that increase hepcidin biosynthesis activate either the BMP or the JAK/STAT3 signaling pathways $(1,2)$. BMP ligands, especially BMP6, and the BMP coreceptor hemojuvelin "sense" serum iron levels (5-7). In iron-replete conditions, the BMP signaling pathway is activated in hepatocytes, leading to downstream SMAD1/5 phosphorylation

Authorship note: Xiang Li and David K. Rhee, as well as Donald B. Bloch and Randall T. Peterson, contributed equally to this work. Kenneth D. Bloch is deceased.

Conflict of interest: The authors have declared that no conflict of interest exists. Submitted: July 16, 2015; Accepted: November 5, 2015.

Reference information: J Clin Invest. 2016;126(1):389-401. doi:10.1172/JCI83831. and an increase in hepcidin gene expression (6). Infections and other inflammatory stimuli (such as IL-6) activate the JAK/STAT3 pathway, which induces hepcidin gene transcription (8-10).

Elevated hepcidin levels cause decreased iron absorption from the gastrointestinal tract and prevent the release of iron from hepatocytes and macrophages, resulting in hypoferremia (1). Conversely, low hepcidin levels cause an increase in iron absorption from the gastrointestinal tract and promote iron release from iron stores, resulting in hyperferremia (1). Persistent low hepcidin levels contribute to the development of iron overload in diseases such as hemochromatosis and $\beta$-thalassemia $(1,11,12)$. Current therapies to treat iron overload in these diseases include phlebotomy and/or iron chelation therapy $(13,14)$. Phlebotomy prevents iron overload in patients with hemochromatosis but cannot be used to treat patients with $\beta$-thalassemia because it exacerbates chronic anemia. Although iron chelators prevent iron overload in patients with hemochromatosis and $\beta$-thalassemia, chelators have side effects, including hepatic and renal toxicity (15). Although iron chelators will likely remain the mainstay of treatment for iron overload disorders, developing additional agents (such as hepcidin inducers or minihepcidins) to prevent iron overload might allow for iron chelators to be administered at lower doses or less frequently. Hence, there is a need to develop additional therapies for the treatment of iron overload disorders. 

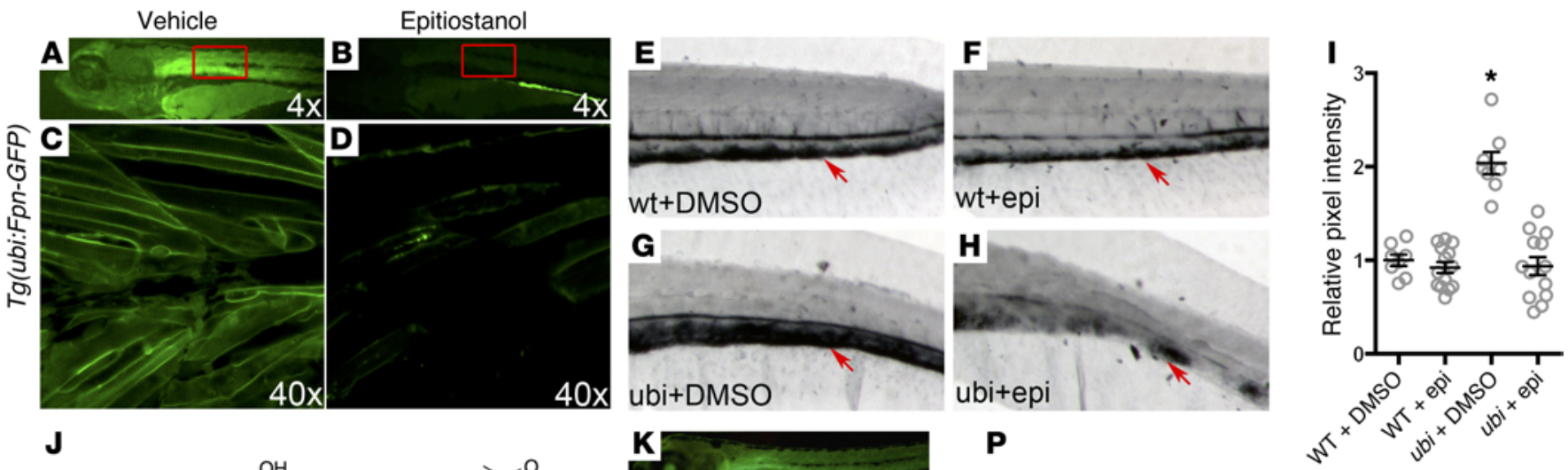

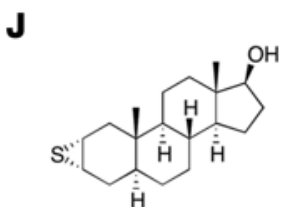

Epitiostanol

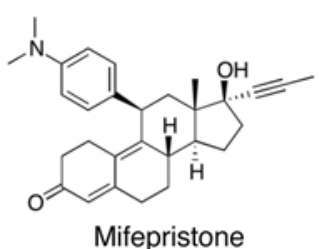

Mifepristone

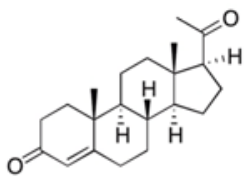

Progesterone

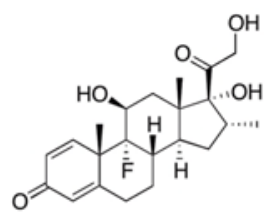

Dexamethasone
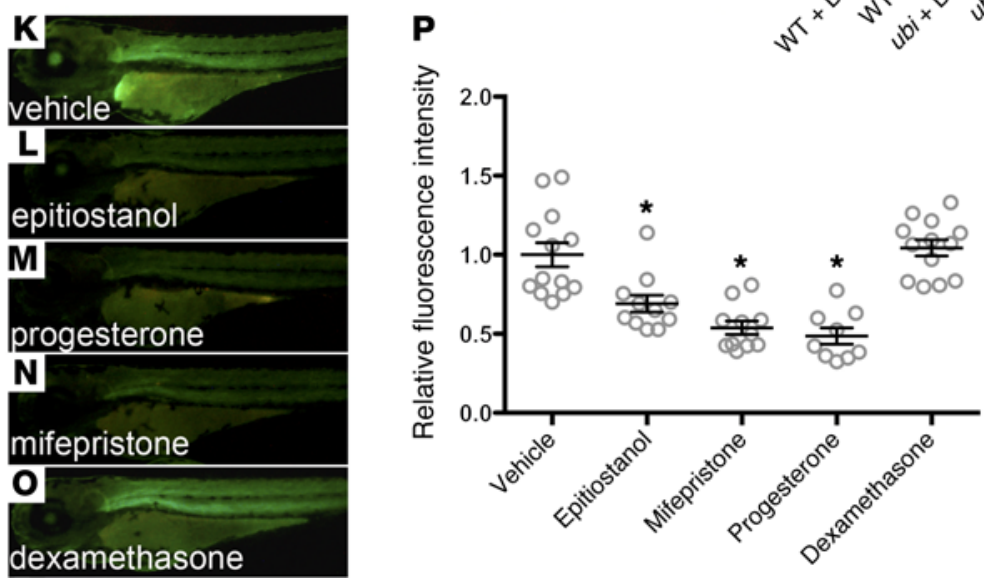

Figure 1. A small-molecule screen in transgenic zebrafish identifies compounds that promote the degradation of ferroportin. (A-D) Fluorescent dissection microscopy and confocal microscopy of Fpn-GFP transgenic zebrafish larvae treated with vehicle (0.1\% DMSO) or $10 \mu \mathrm{M}$ epitiostanol (dissolved in DMSO). Three-day-old transgenic embryos were soaked in E3 buffer containing either vehicle or epitiostanol for 12 hours before imaging (original magnification, $\times 4$ [A and $\mathbf{B}$ ]; $\times 40$ [C and $\mathbf{D}]$ ). High-magnification images of boxed regions in $\mathbf{A}$ and $\mathbf{B}$ are shown in $\mathbf{C}$ and $\mathbf{D}$, respectively. (E-I) Iron staining of wildtype and ferroportin transgenic fish (ubi) treated with DMSO or epitiostanol (epi). Three-day-old embryos were treated with DMSO or $10 \mu \mathrm{M}$ epitiostanol dissolved in DMSO for 12 hours and then fixed. Enhanced Prussian blue staining was then performed on each embryo. Arrows indicate iron deposition in the caudal hematopoietic tissue (original magnification, $\times 8$ [E-H]). Iron staining was quantified by ImageJ based on the pixel intensity, as shown in I and described in the Methods. Results are expressed as mean \pm SEM, ${ }^{*} P<0.001$ ubi plus DMSO ( $n=8$ ), compared with wild-type plus DMSO ( $n=8$ ), wildtype plus epitiostanol $(n=14)$, or ubi plus epitiostanol $(n=13)$, ANOVA. (J) Chemical structure of some compounds that were tested in this screen. (K-P) Fluorescent microscopy (original magnification, $\times 4$ ) of ferroportin transgenic fish treated with the steroid compounds listed in J. Three-day-old transgenic embryos were treated with vehicle (DMSO, $n=13), 10 \mu \mathrm{M}$ epitiostanol dissolved in DMSO $(n=11), 10 \mu \mathrm{M}$ mifepristone $(n=11), 10 \mu \mathrm{M}$ progesterone ( $n=9)$, or $10 \mu \mathrm{M}$ dexamethasone $(n=13)$ for 12 hours, respectively. $(\mathbf{P})$ The intensity of fluorescent staining was quantified for each treatment group and is further described in the Methods. Results are expressed as mean \pm SEM, ${ }^{*} P<0.01$, compared with the vehicle control, ANOVA.

There is considerable interest in developing therapies that increase hepcidin levels (and decrease iron levels) to treat iron overload (16-19). Synthetic hepcidin peptides decrease iron levels in mice and have been developed for the treatment of hemochromatosis, although they are not currently in clinical use $(16,18)$. RNAi-based strategies have been used to increase hepcidin levels in mice by decreasing the expression of TMPRSS6, a negative regulator of hepcidin gene expression (19). The administration of siRNAs directed against TMPRSS6 increased hepcidin levels and reduced iron overload in a mouse model of $\beta$-thalassemia (19). Thus, modulating hepcidin levels may provide new therapeutic approaches for preventing iron overload in patients who may not tolerate traditional therapies.

To identify novel biological pathways important for the regulation of iron metabolism and discover potential drug candidates for the treatment of iron overload disorders, we performed a chemical screen in zebrafish to identify novel small molecules that could decrease ferroportin protein levels. Chemical libraries consisting of bioactive small molecules, including FDA-approved drugs, were used in this screen, with the hope of facilitating the rapid clinical implementation of new therapies for iron overload diseases. This screen led to the identification of a class of structurally similar steroids, including epitiostanol, progesterone, and mifepristone, which decrease ferroportin protein levels by increasing hepcidin gene expression. These hepcidin-inducing steroids (HISs) increase hepcidin biosynthesis by a distinct signaling pathway involving a membrane-bound progesterone receptor, progesterone receptor membrane component-1 (PGRMC1).

\section{Results}

A high-throughput chemical screen in zebrafish leads to the identification of small molecules that decrease ferroportin protein levels. Ferroportin is the sole exporter of iron in vertebrates and thus has an important role in systemic iron homeostasis $(1,2)$. To discover novel pathways important for the regulation of iron metabolism in vivo and new therapeutic targets for the treatment of iron overload 
Zebrafish
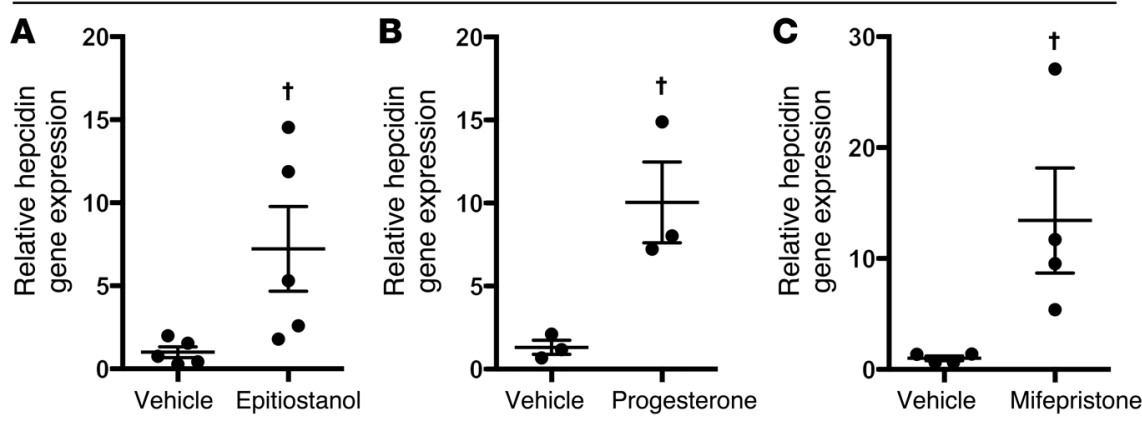

HepG2
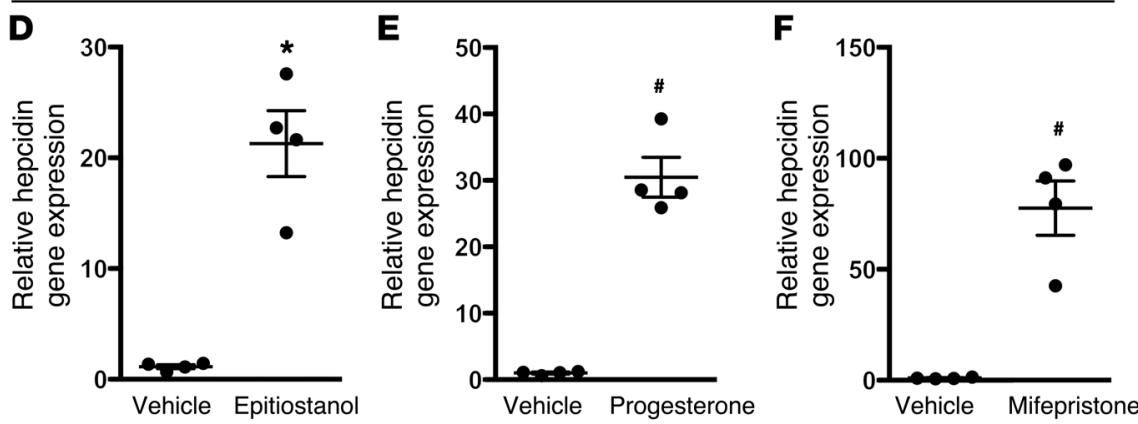

Figure 2. Epitiostanol, progesterone, and mifepristone increase hepcidin gene expression. (A-C) qPCR for hepcidin gene expression in zebrafish larvae treated with vehicle (DMSO) or $10 \mu \mathrm{M}$ epitiostanol (dissolved in DMSO) ( $n=5$ per group), vehicle (ethanol) or $5 \mu \mathrm{M}$ progesterone ( $n=3$ per group), and vehicle (ethanol) or $5 \mu \mathrm{M}$ mifepristone $(n=4$ per group), respectively. Three-day-old zebrafish larvae were treated with each steroid for 12 hours and then lysed for analysis. ${ }^{\dagger} P<0.05$ compared with vehicle treated, 2-tailed $t$ test. (D-F) qPCR for hepcidin gene expression in human HepC2 cells treated with vehicle (DMSO) or $30 \mu \mathrm{M}$ epitiostanol dissolved in DMSO (for 24 hours), vehicle (ethanol) or $30 \mu \mathrm{M}$ progesterone (for 8 hours), and vehicle (ethanol) or $30 \mu \mathrm{M}$ mifepristone (for 8 hours), respectively. Results are expressed as mean $\pm \mathrm{SEM},{ }^{*} P<0.01$ compared with vehicle treated, ${ }^{\#} P<0.001$ compared with vehicle treated, 2 -tailed $t$ test $(n=4$ per group for HepG2 cells). ( $\mathbf{G}$ and $\mathbf{H}$ ) qPCR for hepcidin gene expression in HepG2 cells pretreated with $10 \mu \mathrm{M}$ actinomycin $D$ for 30 minutes and then incubated with vehicle (ethanol), $30 \mu \mathrm{M}$ progesterone, or 30 $\mu \mathrm{M}$ mifepristone for 8 hours. Results are expressed as mean $\pm \mathrm{SEM},{ }^{\#} P<0.001$ compared with control vehicle treated, ${ }^{\#} P<0.001$ compared with control cells treated with progesterone or mifepristone, 2 -way ANOVA ( $n=4$ per group).
G

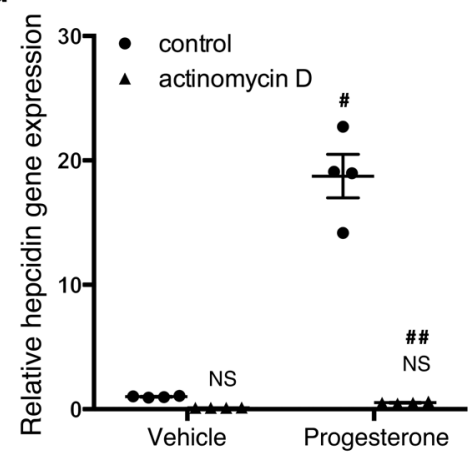

H

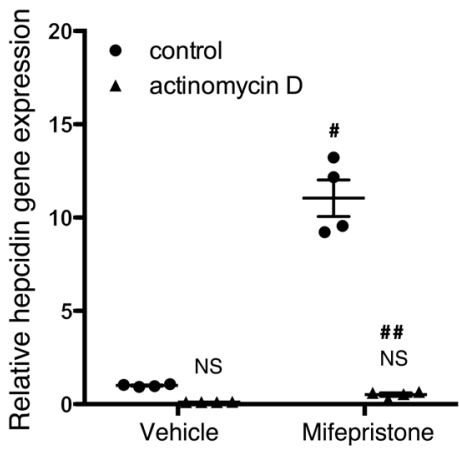

disorders, a chemical screen was performed in zebrafish to identify small molecules that induce ferroportin degradation. A transgenic zebrafish line was developed that expresses mouse ferroportin fused to GFP (Fpn-GFP) under the control of the zebrafish ubi promoter [Tg(ubi:Fpn-GFP)] (Figure 1A). Microscopic examination of transgenic embryos revealed that fluorescence localized to cell membranes throughout the embryo. Transgenic fish were arrayed in 96-well plates, and 3,120 bioactive small molecules from 2 libraries were screened to identify compounds that degrade Fpn-GFP. The degradation of Fpn-GFP was indicated by a loss of, or decrease in, green fluorescence. Epitiostanol was identified in this screen as a small molecule that decreased Fpn-GFP levels in transgenic zebrafish (Figure 1B). Confocal microscopy confirmed a decrease in Fpn-GFP levels from the cell membranes of transgenic zebrafish exposed to epitiostanol (Figure 1, C and D).

To determine whether the apparent epitiostanol-induced loss of Fpn-GFP affected ferroportin activity, we performed iron staining in zebrafish to detect changes in systemic iron levels. The overexpression of ferroportin in transgenic fish caused iron overload in the caudal hematopoietic tissue approximately 3 days after fertilization, as shown by whole-mount iron staining (Figure 1, E and $G)$. Treatment of transgenic fish with epitiostanol $(10 \mu \mathrm{M})$ for 12 hours rescued the iron overload phenotype (Figure 1, E-I).

Epitiostanol is structurally similar to other steroid hormones. To determine whether a broad spectrum of steroid molecules could decrease Fpn-GFP levels, transgenic zebrafish were exposed to other steroid molecules, including estrogen, testosterone, dexamethasone, progesterone, and mifepristone (Figure 1, J-O, and data not shown). Of all steroids tested, only progesterone and mifepristone reduced the GFP signal in the transgenic fish to an extent that was comparable to that seen with epitiostanol (Figure $1, \mathrm{~K}-\mathrm{P})$. Thus, a high-throughput chemical screen in zebrafish led to the identification of 3 structurally similar compounds that promote the degradation of ferroportin in vivo.

Epitiostanol, progesterone, and mifepristone increase hepcidin gene expression. Epitiostanol might decrease Fpn-GFP in transgenic zebrafish by decreasing ferroportin mRNA stability or by enhancing protein degradation. To consider the former possibil- 
A

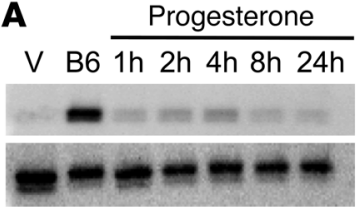

C

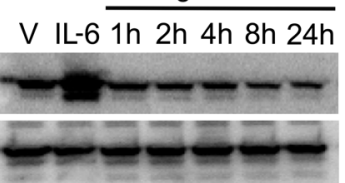

E

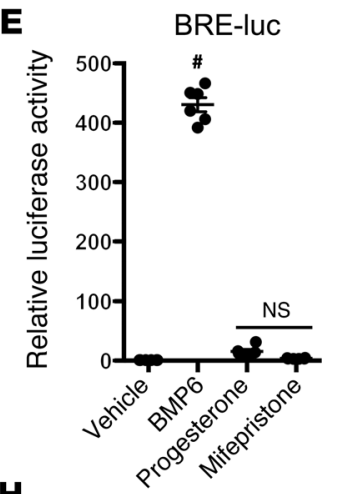

$\mathbf{H}$

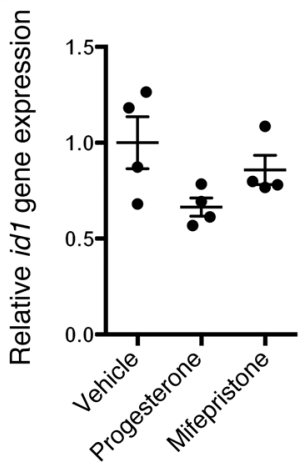

B V B6 $\frac{\text { Mifepristone }}{1 \mathrm{~h} 2 \mathrm{~h} 4 \mathrm{~h} 8 \mathrm{~h} 24 \mathrm{~h}}$

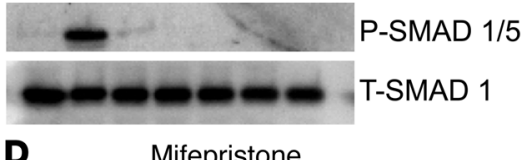

\section{Mifepristone}

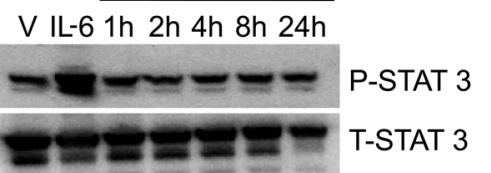

$\mathbf{F}$

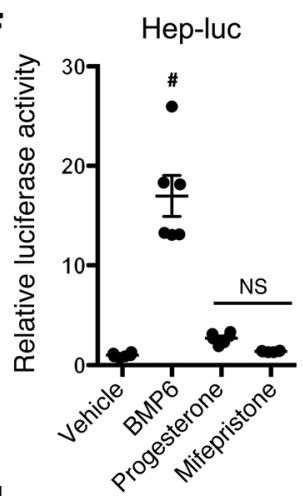

I

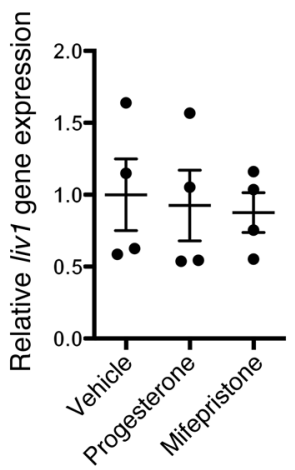

Figure 3. HISs do not activate the BMP or STAT3 signaling pathway. (A and B) SMAD1/5 phosphorylation (P-SMAD 1/5) and total SMAD1 (T-SMAD 1) levels in HepG2 cells exposed to vehicle (V) or BMP6 (20 ng/ml) for 2 hours and to progesterone $(30 \mu \mathrm{M})$ or mifepristone $(30 \mu \mathrm{M})$ for up to 24 hours. (C and D) STAT3 phosphorylation and total STAT3 levels in HepG2 cells exposed to vehicle or IL-6 $(100 \mathrm{ng} / \mathrm{ml})$ for 2 hours and to progesterone $(30 \mu \mathrm{M})$ or mifepristone $(30 \mu \mathrm{M})$ for up to 24 hours. Representative Western blots from 3 independent experiments are shown in A-D. (E) Treatment of HepG2 cells with BMP6 $(20 \mathrm{ng} / \mathrm{ml})$ for 24 hours increased Id1 promoter activity (BRE-luc), while treatment with progesterone (30 $\mu \mathrm{M})$ or mifepristone (30 $\mu \mathrm{M})$ had no effect on Id1 promoter activity. (F and $\mathbf{G})$ Treatment of HepG2 cells for 24 hours with (F) BMP6 $(20 \mathrm{ng} / \mathrm{ml})$ or (G) IL-6 $(100 \mathrm{ng} / \mathrm{ml})$ increased hepcidin promoter activity nearly 15 -fold and 5 -fold, respectively, as measured by the hepcidin luciferase promoter assay (Hep-luc), while treatment of HepC2 cells with progesterone $(30 \mu \mathrm{M})$ or mifepristone $(30 \mu \mathrm{M})$ did not affect hepcidin promoter activity. Results in E-G are expressed as mean \pm SEM, ${ }^{\#} P<0.001$ compared with vehicle treated, ANOVA ( $n=6$ per group). ( $\mathbf{H}$ and $\mathbf{~}$ ) qPCR for id1 and liv1 gene expression in zebrafish. Three-dayold wild-type zebrafish larvae were treated with progesterone $(5 \mu \mathrm{M})$ or mifepristone $(5 \mu \mathrm{M})$ for 12 hours and then lysed prior to qPCR analysis. Results are expressed as mean \pm SEM ( $n=4$ per group). ity, wild-type zebrafish embryos were treated with epitiostanol, and ferroportin mRNA levels were measured by quantitative RT-PCR (qPCR). Epitiostanol did not decrease ferroportin mRNA levels (Supplemental Figure 1; supplemental material available online with this article; doi:10.1172/JCI83831DS1), suggesting that epitiostanol decreases ferroportin protein levels by a posttranscriptional mechanism. Because hepcidin binds to ferroportin and induces its degradation (3), we considered the possibility that epitiostanol, progesterone, and mifepristone induce hepcidin expression. Hepcidin gene expression was measured in wild-type zebrafish embryos exposed to each of the 3 steroids. Compared with treatment with vehicle alone, each steroid increased hepcidin gene expression in zebrafish (Figure 2, A-C). To test whether the HISs epitiostanol, progesterone, and mifepristone had a similar effect on mammalian cells, additional studies were performed using the human hepatoma cell line HepG2. HepG2 cells were incubated with HISs at various time points. Epitiostanol maximally increased hepcidin mRNA levels after 24 hours, while progesterone and mifepristone maximally increased hepcidin gene expression after 8 hours (Figure 2, D-F, and data not shown).
Other steroid compounds that failed to reduce Fpn-GFP levels in zebrafish, including testosterone, dexamethasone, estrogen, and hydrocortisone, had no effect on hepcidin gene expression in HepG2 cells (Supplemental Figure 2).

Of the HISs, progesterone and mifepristone are well-characterized molecules in widespread clinical use. Therefore, progesterone and mifepristone were used for the subsequent experiments. To test whether the increase in hepcidin gene expression by HISs was dependent upon transcriptional activation, HepG2 cells were incubated with actinomycin D and then exposed to HISs. Actinomycin D treatment blocked the ability of the HISs to induce hepcidin gene expression (Figure 2, G and H), suggesting that HISs may increase hepcidin gene expression through transcriptional activation.

HIS-induced hepcidin gene expression is not mediated by BMP or JAK/STAT3 signaling pathways. Various stimuli increase hepcidin transcription through activation of either the BMP or the JAK/ STAT3 signaling pathways (1). For example, iron activates the canonical BMP signaling pathway in hepatocytes, leading to phosphorylation of SMAD1/5 and an increase in hepcidin gene expres- 

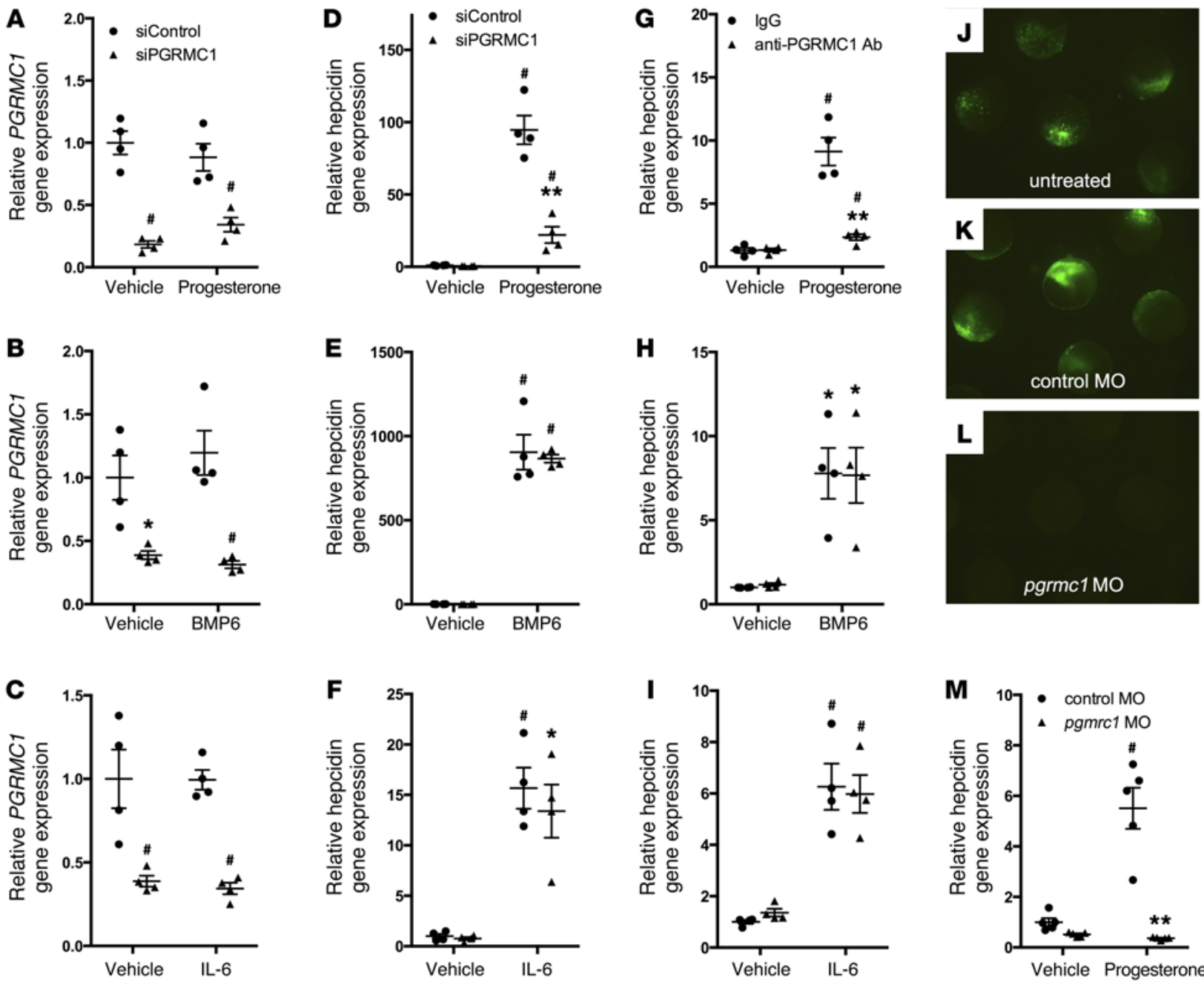

Figure 4. PGRMC1 is required for progesterone, but not BMP6 or IL-6, to increase hepcidin gene expression. (A-C) qPCR for PGRMC1 gene expression in HepG2 cells transfected with scrambled siRNAs (siControl) or siRNAs directed against PGRMC1 (siPGRMC1) and exposed to vehicle, (A) progesterone (30 $\mu \mathrm{M}$ for 8 hours), (B) BMP6 $\left(20 \mathrm{ng} / \mathrm{ml}\right.$ for 3 hours), or (C) IL-6 $\left(100 \mathrm{ng} / \mathrm{ml}\right.$ for 3 hours. ${ }^{*} P<0.01,{ }^{\#} P<0.001$ compared with siControl cells treated with vehicle, 2-way ANOVA ( $n=4$ per group). (D-F) qPCR for hepcidin gene expression in HepG2 cells transfected with siControl or siPCRMC1 siRNAs and treated with vehicle, (D) progesterone, (E) BMP6, or (F) IL-6 (identical concentrations and incubation times as above). (G-I) qPCR for hepcidin gene expression in HepG2 cells preincubated with control IgG or anti-PGRMC1 antibodies (PGRMC1 Ab) for 3 hours and then exposed to vehicle or (G) progesterone, (H) BMP6, or (I) IL-6 (identical concentrations and incubation times as above). ${ }^{*} P<0.001,{ }^{*} P<0.01$, compared with siControl cells (D-F) or IgG-treated cells (G-I) exposed to vehicle; ${ }^{* *} P<0.001$ compared with siControl cells (D) or IgG-treated cells (G) exposed to progesterone, 2-way ANOVA ( $n=4$ per group). (J-L) A plasmid containing the pgrmc1 morpholino oligonucleotide target site upstream of an EGFP coding sequence was injected (J) alone, (K) with control morpholino oligonucleotides (control MO), or (L) with pgrmc1 morpholino oligonucleotides (pgrmc1 MO) into 1-cell stage zebrafish embryos, and fluorescence was examined after 8 hours (original magnification, $\times 2.5$ ). (M) qPCR for hepcidin gene expression in 3-day-old wild-type zebrafish embryos injected with control morpholino oligonucleotides or pgrmc1 morpholino oligonucleotides and treated with vehicle or progesterone $(5 \mu \mathrm{M}$ for 12 hours). \# $P<0.001$ compared with control morpholino oligonucleotides embryos treated with vehicle, ${ }^{* *} P<0.001$ compared with control morpholino oligonucleotides embryos treated with progesterone, 2 -way ANOVA ( $n=5$ per group). qPCR results expressed as mean \pm SEM.

sion (1). Inflammatory stimuli, such as IL-6, stimulate the JAK/ STAT3 pathway, leading to STAT3 phosphorylation and increased hepcidin biosynthesis (1). To test whether HISs might induce hepcidin gene expression by activating either the BMP or JAK/STAT3 pathway, HepG2 cells were treated with BMP6, IL-6, or HISs, and cell lysates were prepared at serial time points. Western blots were performed with antibodies directed against either phosphorylated SMAD1/5 or phosphorylated STAT3 to assess the activation of the BMP or JAK/STAT3 signaling pathway, respectively. BMP6 increased phosphorylation of SMAD1/5 in HepG2 cells, while cells treated with HISs showed either no increase or, in the case of progesterone-treated cells, only a slight increase in SMAD1/5 phosphorylation compared with cells treated with vehicle alone (Figure 3 , A and B). HepG2 cells treated with IL-6 showed an increase in STAT3 phosphorylation, while cells incubated with HISs had no increase in STAT3 phosphorylation (Figure 3, C and D).

As an additional approach to test whether progesterone or mifepristone might activate the BMP signaling pathway in hepatocytes, HepG2 cells were transfected with a luciferase construct in which a BMP-responsive element from the $I d 1$ promoter drives 
A

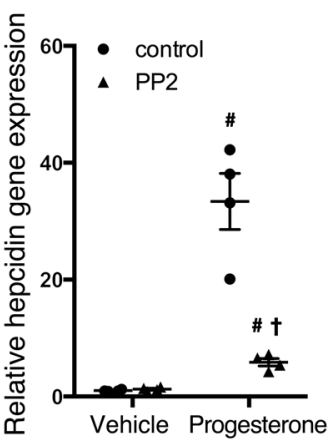

B

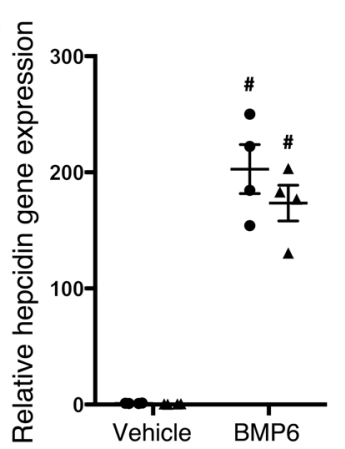

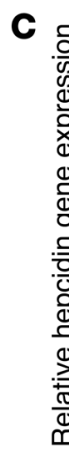

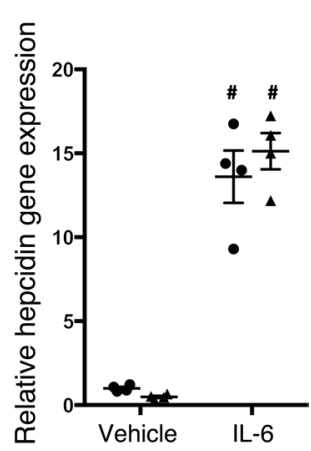

Figure 5. Progesterone, but not BMP6 or IL-6, requires SFK activity to increase hepcidin gene expression. qPCR for hepcidin gene expression in HepG2 cells serum starved for 18 hours and then preincubated with the SFK inhibitor PP2 $(10 \mu \mathrm{M})$ or control (DMSO) for 30 minutes and then exposed to (A) progesterone (30 $\mu \mathrm{M}$ for 8 hours), (B) BMP6 (20 ng/ml for 3 hours), or (C) IL-6 (100 ng/ml for 3 hours). ${ }^{\#} P<0.001$ compared with control treated cells exposed to vehicle, ${ }^{\dagger} P<0.001$ compared with control treated cells exposed to progesterone, 2-way ANOVA ( $n=4$ per group). All results expressed as mean $\pm \mathrm{SEM}$. the expression of luciferase (BRE-luc) (20). Treatment with BMP6 increased Id 1 promoter activity in HepG 2 cells transfected with the BRE-luc construct but neither progesterone nor mifepristone increased $I d 1$ promoter activity, confirming that neither compound activates the BMP signaling pathway in HepG2 cells (Figure 3E). The proximal promoter of the hepcidin gene contains both BMP-responsive elements and a STAT3-binding site $(10,21)$. Consistent with the results reported by other investigators (22), we observed an increase in hepcidin promoter activity when HepG2 cells were transfected with a luciferase construct that contains $2.7 \mathrm{~kb}$ of the proximal hepcidin promoter (hepluc) and then treated with BMP6 or IL-6 (Figure 3, F and G). In contrast, there was no increase in hepcidin promoter activity in hep-luc-transfected cells treated with HISs (Figure 3, F and G). These results suggest that HISs do not increase hepcidin gene expression by activating BMP or JAK/STAT3 signaling and that HISs use a regulatory element that is outside the $2.7-\mathrm{kb}$ proximal hepcidin promoter in the hep-luc construct to regulate the transcription of hepcidin.

To confirm the finding that HISs do not activate BMP or JAK/ STAT3 pathways in an in vivo system, zebrafish embryos were exposed to HISs and qPCR was performed to measure mRNA levels of the BMP downstream gene $i d 1$ and the Jak/Stat 3 downstream gene liv1. There was no increase in $i d 1$ and liv1 gene expression in wild-type zebrafish embryos exposed to HISs compared with that in zebrafish embryos treated with vehicle alone (Figure 3, $\mathrm{H}$ and I). These combined findings indicate that HISs regulate hepcidin gene expression by a signaling pathway that is distinct from the BMP and JAK/STAT3 pathways.

PGRMC1 is required by HISs to increase hepcidin biosynthesis. Both progesterone and the classical progesterone receptor antagonist mifepristone increased hepcidin gene expression in vitro and in vivo, suggesting that the progesterone (nuclear hormone) receptor is not the relevant target of these steroids (progesterone and mifepristone have opposite effects on the classical progesterone receptor). In addition, the HISs induced hepcidin in HepG2 cells, but the classical progesterone receptor was not expressed in HepG 2 cells, as determined by quantitative PCR using specific TaqMan probes (data not shown), a finding which has also been reported by others (23). Because the membrane-bound progesterone receptor PGRMC1 is expressed in HepG2 cells and zebrafish and is activated by progesterone $(24,25)$, we considered the possibility that PGRMC1 might mediate the effects of HISs on hepcidin gene expression. To determine whether PGRMC1 is nec- essary for the hepcidin-inducing activity of HISs, PGRMC1 transcripts were depleted in HepG2 cells using siRNAs, and cells were then exposed to HISs. Successful knockdown of PGRMC1 transcripts in HepG2 cells treated with vehicle, HISs, BMP6, or IL-6 was confirmed by qPCR (Figure 4, A-C, and Supplemental Figure 3A). Knockdown of PGRMC1 in HepG2 cells caused a significant reduction in HIS-induced hepcidin gene expression (Figure 4D and Supplemental Figure 3B). Depleting PGRMC1 in HepG2 cells did not alter the ability of either BMP6 or IL- 6 to increase hepcidin gene expression (Figure 4, E and F), showing that the BMP and JAK/STAT3 signaling pathways function independently of PGRMC1 to regulate hepcidin gene transcription. In these experiments, cells were maintained in serum-free media for 52 hours prior to sample collection (see the Methods for full details). This duration of serum starvation was necessary to allow the cells time to fully recover after transfection with siRNA. The absence of sera did not appear to alter cell morphology or viability, as assessed by microscopy. In addition, these cells still maintained the ability to increase hepcidin gene expression in response to BMPs and IL-6, suggesting that, despite the absence of sera, the hepcidin-regulatory mechanisms remained intact.

As an alternate approach to assessing the role of PGRMC1 in the regulation of hepcidin gene expression by HISs, HepG 2 cells were incubated with a polyclonal antibody directed against the extracellular domain of PGRMC1. Previous investigators used this antibody to block progesterone signaling through PGRMC1 (26). Incubating HepG2 cells with an antibody directed against the extracellular domain of PGMRC1, but not a species-matched control IgG antibody, attenuated the ability of HISs to induce hepcidin gene expression in HepG2 cells (Figure 4G and Supplemental Figure 3C). In contrast, the PGRMC1-blocking antibody did not alter the ability of BMP6 and IL-6 to increase hepcidin biosynthesis (Figure 4, H and I). Note that in cells treated with either anti-PGRMC1 or isotype-matched antibody, the relative induction of hepcidin by HISs, BMP6, or IL-6 was decreased compared with the effects of these agonists on cells that were not exposed to antibodies. This difference is likely due to nonspecific blocking effects of the antibodies and/or differences in the length of serum deprivation prior to the addition of HISs, BMP6, or IL- 6 between experiments (cells were serum starved for 18 hours for the antibody-blocking experiments and for 52 hours for the experiments with siRNAs, as described above and in the Methods). Pretreatment of HepG2 cells with anti-PGRMC1 antibodies specifically blocked the HIS-mediated, but not the BMP6- or IL-6-mediated, 

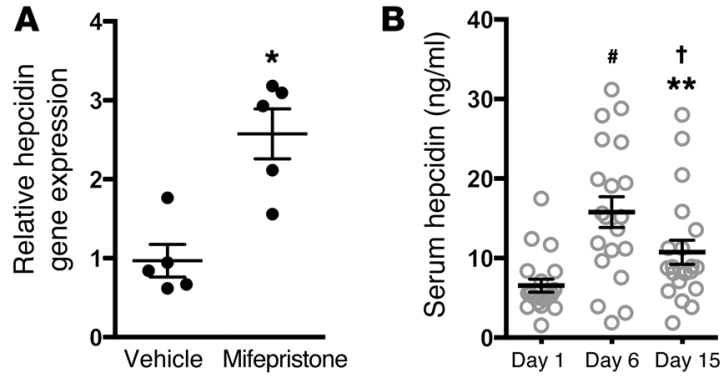

Figure 6. HISs increase hepcidin biosynthesis in mice and humans. (A) qPCR of hepcidin gene expression in mouse liver 10 hours after injection with vehicle or mifepristone. ${ }^{*} P<0.05,2$-tailed $t$ test ( $n=5$ per group). (B) Serum hepcidin levels in women prior to progesterone treatment (day 1) or after receiving progesterone daily (day 6 and day 15 ) during a standard in vitro fertilization protocol using FET. ${ }^{*} P<0.001$ compared with day 1 sample, ${ }^{\dagger} P<0.01$ compared with day 1 sample, ${ }^{* *} P<0.01$ compared with day 6 sample, paired $t$ test with Bonferroni adjustment for multiple comparisons ( $n=20$ per group). All results expressed as mean \pm SEM.

induction of hepcidin gene expression. These findings further demonstrate that HISs require PGRMC1 to regulate hepcidin gene expression. In addition, these studies again demonstrate that BMP6 and IL-6 regulate hepcidin biosynthesis by a mechanism that does not involve PGRMC1.

To determine the role of PGRMC1 in the HIS-mediated induction of hepcidin in vivo, $\mathrm{pgrmc1}$ transcripts were depleted in zebrafish using antisense morpholino oligonucleotides, and zebrafish were then exposed to progesterone or mifepristone. Knockdown of pgrmc1 transcripts in zebrafish was confirmed using an antisense morpholino efficiency test (Figure 4, J-L, and ref. 27). Knockdown of pgrmc1 in zebrafish blocked the ability of HISs to increase hepcidin gene expression (Figure 4M and Supplemental Figure 3D). These combined data indicate that PGRMC1 is required for HISmediated induction of hepcidin both in vitro and in vivo.

Inhibition of SRC family kinases abolishes the induction of hepcidin by HISs. PGRMC1 has been implicated in modulating the activity of different downstream signaling pathways, including the SRC family kinase (SFK), PKA, PI3 kinase, and PKC pathways $(24,28,29)$. Systematically testing each potential downstream pathway, using specific pharmacological inhibitors, demonstrated that the SFK pathway was required for the effects of HISs on hepcidin gene expression. Pretreatment of HepG2 cells with the SFK chemical inhibitor PP2 (30) blocked the ability of HISs, but not BMP6 or IL-6, to increase hepcidin biosynthesis (Figure 5 and Supplemental Figures 4 and 5). Interestingly, pretreatment of cells with inhibitors of the PI3 kinase pathway, including LY294002 or wortmannin, potentiated the induction of hepcidin by progesterone (Supplemental Figure 5, B and C), raising the possibility that the PI3 kinase pathway might suppress the induction of hepcidin by HISs. Pretreatment of cells with PP3 (31), a negative control for PP2, did not block the ability of HISs to increase hepcidin gene expression (data not shown). These data further demonstrate that HISs induce hepcidin gene expression through a mechanism that is distinct from the previously identified hepcidin-regulatory mechanisms and that PGRMC1 and its downstream SFK signaling cascade are essential components of this pathway.
HISs increase hepcidin biosynthesis in mice and humans. We sought to determine whether the HIS-PGRMC1-mediated effects on hepcidin biosynthesis were conserved in mice. Mifepristone was more potent than progesterone in inducing hepcidin gene expression in zebrafish and HepG2 cells (data not shown). Therefore, to maximize the possibility of observing an effect of HISs on hepcidin biosynthesis in vivo, mifepristone was tested for its ability to increase hepatic hepcidin gene expression in mice. Tenweek-old C57BL/6 wild-type female mice were fed an iron-deficient diet for 14 days. Mifepristone or vehicle was subsequently administered by intraperitoneal injection. Mifepristone was not soluble in aqueous solution and, therefore, was dissolved in a nontoxic solution containing diethylene glycol monoethyl ether, Cremophor EL, and phosphate-buffered saline in a 1:1:3 ratio, as described by others (32). Mice were sacrificed 10 hours after injection, and liver tissue was harvested. Hepcidin gene expression increased approximately 2.5 -fold in the livers of mice injected with mifepristone compared with that in mice injected with vehicle alone ( $2.57 \pm 0.31$ vs. $0.96 \pm 0.207, P<0.05$; Figure $6 \mathrm{~A})$.

We next sought to determine whether HISs might increase hepcidin biosynthesis in humans. During the menstrual cycle, progesterone initiates the endometrial transition from the proliferative stage to the secretory stage, thereby establishing uterine receptivity for embryo implantation (33). In addition to promoting embryo implantation, endogenous progesterone is also required to maintain a pregnancy. During in vitro fertilization with frozen embryo transfer (FET), exogenous progesterone is administered to prepare the endometrium for embryo implantation and to support early pregnancy (33). For this procedure, women take estrogen orally twice daily, starting on the second day of their menstrual cycle. When the endometrium proliferates to a thickness of greater than $7 \mathrm{~mm}$, exogenous progesterone $(50 \mathrm{mg}$ intramuscular injection daily) is administered until a negative pregnancy test is obtained or throughout the first 8 weeks of pregnancy. To investigate whether HISs increase hepcidin biosynthesis in humans, serum hepcidin levels were measured in 20 women who were given progesterone during in vitro fertilization with FET and did not become pregnant. Serum samples from nonpregnant patients were analyzed to exclude any confounding effects that human chorionic gonadotropin hormone (produced by the placenta after implantation) might have on endogenous progesterone synthesis or hepcidin biosynthesis. Blood samples were collected immediately prior to progesterone injection (day 1), on the day of FET (day 6 of progesterone treatment), and on day 15 of progesterone treatment. Prior to exogenous progesterone administration (day 1), the average serum progesterone level in these patients was $0.308 \mathrm{ng} / \mathrm{ml}$, which increased to an average of $24.6 \mathrm{ng} / \mathrm{ml}$ on day 6 of progesterone treatment and to $30.25 \mathrm{ng} / \mathrm{ml}$ on day 15 of progesterone treatment (Supplemental Figure 6). Serum hepcidin levels increased after the administration of progesterone. There was nearly a 3-fold increase in serum hepcidin levels on day 6 of progesterone treatment and nearly a 2 -fold increase in serum hepcidin levels on day 15 of progesterone treatment compared with samples collected prior to progesterone therapy (day 1) (Figure $6 \mathrm{~B})$. When serum hepcidin levels were measured in these patients using a different ELISA kit (Peninsula Laboratories), which has been used by several other investigators (formerly distributed by 
Bachem and now distributed by Peninsula Laboratories), similar results were obtained (Supplemental Figure 7 and refs. 34-37). These results indicate that HISs can increase hepcidin biosynthesis in humans as well as mice and zebrafish. These results suggest that the HIS-PGRMC1-mediated regulation of hepcidin is evolutionarily conserved.

\section{Discussion}

The hepcidin-ferroportin axis is the central regulator of iron homeostasis in vertebrates $(1,2)$. Perturbations in hepcidin-ferroportin signaling can cause profound shifts in iron levels associated with anemia or hemochromatosis. For this reason, there has been great interest in elucidating the mechanisms that regulate hepcidin-ferroportin signaling and discovering potential pharmacological targets that modulate this axis to treat diseases of abnormal iron metabolism.

The majority of stimuli that increase hepcidin biosynthesis, including increased serum iron and inflammation, do so by activating either the BMP signaling pathway or the JAK/STAT3 pathway. Protein and small-molecule antagonists of the BMP signaling pathway can decrease hepcidin levels and improve anemia in mouse models of the anemia of inflammation (AI) (38-40). These studies demonstrate that the BMP signaling pathway can be modulated by small molecules to decrease hepcidin levels and improve anemia in patients with AI. Small molecules capable of inducing hepcidin could also be of great therapeutic benefit to patients with hemochromatosis or other disorders complicated by iron overload, such as $\beta$-thalassemia. Unfortunately, there has been limited progress in identifying small molecules that increase hepcidin biosynthesis.

By using a phenotype-based chemical screen in zebrafish, we identified a pathway that regulates hepcidin biosynthesis independent of the BMP and JAK/STAT3 pathways. The steroid molecules identified in this screen decrease Fpn-GFP levels by increasing the biosynthesis of hepcidin. HISs increase hepcidin levels by a mechanism that requires a membrane-bound progesterone receptor, PGRMC1. Depletion of PGRMC1 in HepG2 cells and zebrafish abrogated the induction of hepcidin by HISs. In addition, polyclonal antibodies directed against the extracellular domain of PGRMC1 blocked the ability of HISs to increase hepcidin biosynthesis in HepG2 cells.

PGRMC1 has been implicated in a variety of biological processes, but its in vivo functions are poorly understood (25). PGRMC1 was initially identified via biochemical purification of a membrane-bound progesterone receptor from porcine liver microsomes (41). PGRMC1 contains a single transmembrane domain and heme-binding site $(24,25,42)$ and has been shown to mediate rapid noncanonical progesterone signaling (43). In vitro cell culture data suggest that PGRMC1 promotes cell survival and that PGRMC1 is required for the antiapoptotic effect of progesterone in human granulosa cells $(44,45)$. In addition, PGRMC1 serves as a necessary cofactor for $\mathrm{P} 450$ proteins to synthesize cholesterol $(46,47)$. Studies in yeast indicate that the yeast ortholog of PGRMC1, damage-associated protein-1 (Dap1), has an important role in iron metabolism (48). Yeast deficient in Dap1 manifest impaired growth on iron-deficient media, although the mechanisms by which Dap1/PGRMC1 regulates iron metabolism in yeast have not yet been fully elucidated (48). Our findings now implicate PGRMC1 in iron homeostasis in vertebrates, with a specific role in regulating hepcidin gene expression.

Although PGRMC1 regulates the activity of several signal transduction pathways, the PGRMC1-mediated induction of hepcidin appears to be dependent on SFK activity, but not PKA, PKC, or PI3 kinase signal transduction pathways. Although SFKs play a necessary role in the PGRMC1-mediated regulation of hepcidin biosynthesis, several important questions regarding PGRMC1/ hepcidin signaling remain to be elucidated. Foremost among these are questions regarding the signaling cascades that are downstream of the SFKs and the regulatory elements required by HISs to increase the transcription of hepcidin. The transcriptional effects of PGRMC1 can be mediated through tcf/lef transcription factors (49). Thus, it will be interesting in the future to determine whether the induction of hepcidin gene expression by PGRMC1 is mediated by SFK-Tcf/Lef signaling.

In addition to the hepcidin-inducing effects of HISs in zebrafish and cultured human hepatocytes, these steroid hormones also increase hepcidin biosynthesis in mammals. When mifepristone was administered to mice there was an increase in hepatic hepcidin gene expression. In addition, serum hepcidin levels increased in women after they were given progesterone as part of a standard in vitro fertilization protocol. These findings suggest that HIS-mediated hepcidin induction is broadly conserved and physiologically relevant.

The kinetics of the PGRMC1-mediated regulation of hepcidin gene expression are somewhat different than those of the BMPmediated regulation of hepcidin biosynthesis. Other investigators have shown that the BMPs stimulate hepcidin gene expression in Hep3B cells within 1 hour and maximally induce hepcidin at 16 hours (50). Progesterone and mifepristone began to stimulate hepcidin gene expression somewhat later, within 4 hours (data not shown). The maximal induction of hepcidin by progesterone and mifepristone occurred at 12 hours, which is similar to the time required for the maximal induction of hepcidin by BMPs. It is unclear why the initial induction of hepcidin by HISs was slower than that of BMPs. It is possible that HISs cause a gradual accumulation of an intracellular second messenger or metabolite, which, after reaching a critical threshold level, triggers downstream signaling pathways that activate hepcidin gene transcription. Future studies to further elucidate the downstream signaling pathways mediated by HISs and PGRMC1 should provide important insights into the kinetics of this hepcidin-regulatory pathway. In addition, it is unclear why epitiostanol maximally induced hepcidin at a later time point than either progesterone or mifepristone. One possible explanation for this finding is that epitiostanol may need to be converted into an active metabolite before it can activate the PGRMC1/hepcidin pathway.

Sex hormones have been previously implicated in the regulation of hepcidin biosynthesis. Estrogen was found to increase hepcidin gene expression in some studies but decrease hepcidin levels in others (51-53). We observed that in HepG2 cells estrogen caused a modest increase in hepcidin gene expression that failed to reach statistical significance (although our study may have been underpowered to observe a significant change in hepcidin levels). Given that other investigators have also observed an increase in 
hepcidin levels in response to estrogen, it is likely that estrogen is a modest hepcidin inducer. Testosterone has also been shown to regulate hepcidin biosynthesis. Several studies have demonstrated that testosterone causes a decrease in hepcidin levels (54, $55)$. The downregulation of hepcidin gene expression by testosterone appears to be due to multiple factors, including testosterone's stimulatory effect on erythropoiesis, modulation of BMP signaling by the androgen receptor, and alterations in epidermal growth factor receptor signaling $(54,55)$. We did not observe an effect of testosterone on hepcidin gene expression in vitro, but the previously mentioned studies primarily assessed the effect of testosterone on hepcidin biosynthesis in vivo or used different experimental conditions in vitro than those used in this study.

The HISs identified in our study do not appear to share a unique chemical structure that would explain why they, as opposed to other steroid molecules, cause a robust increase in hepcidin gene expression. Epitiostanol, progesterone, and mifepristone are similar to other sex steroid hormones in their chemical structure and use cholesterol as a core structural backbone. Structure-activity relationship assays may help to provide insights into the chemical components unique to HISs that may mediate their hepcidin-inducing activity.

Two outstanding questions are what physiological role PGRMC1 may play in normal iron homeostasis and how this hepcidin-regulatory pathway might contribute to the observed differences in iron homeostasis between males and females. Other investigators have shown that hepcidin levels are higher in female rats and mice when compared with those in males (56). Perhaps progesterone (in addition to other factors) might account for the higher hepcidin levels observed in females in these studies. However, in contrast to studies in rodents, human serum hepcidin levels appear to be lower in women of certain age groups when compared with those in men and higher in other age groups $(57,58)$. How the PGRMC1-hepcidin axis might contribute to the differences in hepcidin levels between men and women in these studies is unclear and warrants further investigation. The observation that progesterone increases hepcidin levels in humans raises the possibility that serum hepcidin levels may increase during high-progesterone states, such as during specific days in a woman's menstrual cycle. Given that the progestational hormone progesterone is also elevated during early pregnancy, it is tempting to speculate about a physiological role for progesterone in regulating serum hepcidin levels during pregnancy. It has been shown that hepcidin levels are highest early in pregnancy and diminish throughout the course of pregnancy (59). PGRMC1 may also serve as a receptor for other endogenous or exogenous ligands besides progesterone, thereby functioning as a node for connecting metabolic requirements and iron availability. Additional studies will be needed to address the physiological role of the PGRMC1 pathway in regulation of hepcidin biosynthesis.

Several groups have demonstrated that increasing hepcidin levels in mice can decrease iron overload in mouse models of $\beta$-thalassemia and hemochromatosis, suggesting that hepcidininducing agents may be useful in the treatment of iron overload diseases $(16,18,19)$. Although chelation therapy will likely remain the mainstay of treatment for patients with iron overload disorders, additional therapeutic agents that increase hepcidin levels, such as HISs, may play an important role in maintenance therapy by limiting further iron accumulation. In addition, the use of these types of drugs might allow for chelators to be administered less frequently or at lower doses. The finding that two widely prescribed medicines, progesterone and mifepristone, increase hepcidin biosynthesis raises the possibility of using these medicines for the treatment of iron overload diseases.

Progesterone has been used for several decades as a contraceptive agent and for hormone replacement therapy. Synthetic progesterone analogs, when used for contraception or hormone replacement therapy, are administered at doses ranging from 2.5 $\mathrm{mg}$ to $100 \mathrm{mg}$ per day (60). These doses are similar to the dose of progesterone administered to women undergoing in vitro fertilization (50 mg daily), during which we observed an increase in serum hepcidin levels. Long-term treatment with progesterone can cause side effects, including depression, fatigue, and peripheral edema (60). Mifepristone has been used as a medicinal abortifacient but is also administered chronically for the treatment of Cushing's syndrome (61). Patients with Cushing's syndrome are treated with mifepristone at doses as high as $1,200 \mathrm{mg}$ per day, which is the equivalent of approximately 10 to $15 \mathrm{mg} / \mathrm{kg}$ per day. Mifepristone's side effect profile includes fatigue, nausea, and hypokalemia. The dose of mifepristone administered for Cushing's disease is approximately 10 times lower than the dose we administered to mice $(100 \mathrm{mg} / \mathrm{kg})$. Future studies will be needed to determine whether mifepristone increases serum hepcidin levels in humans at these lower doses. Future studies will also be needed to address whether chronic treatment with progesterone or mifepristone can prevent iron overload in iron overload disorders and whether the side effect profiles of these drugs are acceptable in this patient population.

In summary, we describe the identification of several HISs that regulate hepcidin biosynthesis in vertebrates from zebrafish to humans. Surprisingly, we found that they do not function via known hepcidin-regulatory pathways. Rather, they regulate hepcidin expression through what we believe to be a novel pathway involving PGRMC1 and SFKs. Our findings provide insights into the regulation of iron homeostasis and also identify potential therapeutic targets and drug candidates for the treatment of iron overload disorders.

\section{Methods}

\section{Reagents}

Progesterone, mifepristone, estradiol, testosterone, dexamethasone, and hydrocortisone were purchased from Sigma-Aldrich and dissolved in ethanol for experiments in HepG2 cells and zebrafish. Epitiostanol was purchased from Prestwick Chemical and dissolved in DMSO for experiments in HepG2 cells and zebrafish. PP2, PP3, G06983, LY294002, Wortmannin, and KT5720 were purchased from Tocris Bioscience and dissolved in DMSO for all experiments. Human BMP6 and IL-6 were purchased from R\&D Systems.

\section{Plasmid design, mRNA synthesis, and microinjection of zebrafish}

The construct for generating $T g(u b i: F p n-G F P)$ zebrafish was engineered by ligating DNA containing the zebrafish $u b i$ promoter upstream of DNA encoding murine ferroportin ( $F p n)$ fused to GFP into the pDestTol2pA2 destination vector from the Tol2kit (Invitrogen) using the gateway strategy. The plasmid encoding Fpn-GFP was a gift from Jerry Kaplan (University of Utah, Salt 
Lake City, Utah, USA), and the Tol2-cloning plasmid was a gift from Caroline Burns (Massachusetts General Hospital). The primers used to amplify the Fpn-GFP cDNA were, FpnGFPattB1F, GGGGACAAGT T TGTACAAAAAAGCAGGCTGTCGCCACCATGACCAAGGCAAGAGATC, and FpnGFPattB2R, GGGGACCACTTTGTACAAGAAAGCTGGGTTTACTTGTACAGCTCGTCC. The $5^{\prime}$ entry clone containing the $u b i$ promoter sequence was provided by Leonard Zon (Boston Children's Hospital, Boston, Massachusetts, USA). The pgrmc1-5' UTR-EGFP construct used for the antisense morpholino efficiency test was generated by subcloning the $5^{\prime}$ UTR and partial coding sequence of zebrafish pgrmc1 into pEGFP-N3 (Clonetech). The primer sequences used to amplify the $5^{\prime}$ UTR and partial coding sequence of zebrafish pgrmc1 were, forward, CGGAATTCAGAATCGAAATGGCTGAAG, and reverse, GGGGTACCGTATCCTTCTTTTTGTCCTTGAC. Tol2 mRNA was synthesized by using an mMESSAGE mMACHINE Kit (Ambion) as previously described (62). For generation of transgenic zebrafish, the plasmids were injected into the cytoplasm of zebrafish embryos at the 1-cell stage along with tol2 transposase mRNA. For the morpholino efficiency tests, plasmids were injected into the cytoplasm of zebrafish embryos at the 1-cell stage together with morpholino oligonucleotides.

\section{Fish maintenance and small-molecule screening}

Wild-type Tubingen AB strain and $T g(u b i: F p n-G F P)$ strains were used. All zebrafish were maintained in the Massachusetts General Hospital fish facility. Three-day-old zebrafish larvae from the $\mathrm{Tg}(u b i$ :Fpn-GFP) strain were plated at 3 fish per well into 96-well plates in E3 buffer. A final concentration of $10 \mu \mathrm{M}$ of each compound from 2 chemical libraries (the Prestwick Chemical Library [Prestwick Chemical] and the Spectrum Collection [MicroSource Discovery]) was directly added to each well. After 12 hours of incubation at $28.5^{\circ} \mathrm{C}$, the fish in each well were scored using a fluorescent dissection microscope (Carl Zeiss). Compounds that caused a loss or decrease of GFP fluorescence in all 3 fish in a well were considered positive hits. A secondary validation of each positive hit was performed by treating larger numbers of transgenic fish with each positive hit compound at different concentrations and then measuring fluorescence.

Measurement of fluorescent intensity in transgenic zebrafish embryos Fluorescence intensity was measured in whole fish by orienting embryos with the head to the left and acquiring images at $\times 4$ magnification. An exact area of $1,388 \times 1,040$ pixels was captured for each image.

Whole-mount zebrafish iron staining and quantification of iron staining DAB-enhanced iron staining was performed on 4-day-old Tg(ubi:FpnGFP) embryos after fixation with 4\% PFA and dehydration with methanol as previously described (63). Prior to staining, PFA-fixed zebrafish embryos were bleached by using a buffer containing $0.8 \% \mathrm{KOH}, 9 \%$ $\mathrm{H}_{2} \mathrm{O}_{2}$, and $0.1 \%$ Tween 20. Iron staining was quantified by measuring the intensity of Prussian blue staining in the caudal hematopoietic tissue of each fish. To ensure that the same region was measured in each fish, all embryos were oriented with the head to the left, and images were acquired using the same magnification $(\times 8)$. Pixel intensity was measured within a region of interest measuring $1,600 \times 1,200$ pixels, extending from the beginning of the caudal hematopoietic tissue near the cloaca to the end of the tail fin.

\section{Morpholinos}

The antisense morpholino oligonucleotides used to inhibit the translation of pgrmc1 were designed and synthesized by Gene Tools LLC (pgrmc1 morpholino oligonucleotides). Standard 25-N antisense morpholino oligonucleotides, used as control morpholinos, were also purchased from Gene Tools LLC (25-N morpholino oligonucleotides).

\section{Microscopy}

For live imaging, transgenic embryos were mounted with low-melting-point agarose at the desired stages. GFP fluorescence images were acquired by a Zeiss Discovery V8 fluorescent stereo microscope $(\times 4)$ and a Zeiss LSM 700 confocal microscope $(\times 40$, water immersion). For iron staining, embryos were mounted with glycerol, and the images were obtained using the Leica WILD M10 stereo microscope using SPOT Advanced software. Quantification of iron staining and GFP fluorescence was based on pixel intensity, as measured by ImageJ software.

\section{Cells and media}

Human hepatoma cells (HepG2 cells) were obtained from SigmaAldrich and cultured in Eagle's Minimum Essential Medium (EMEM) supplemented with $10 \%$ fetal bovine serum, penicillin, streptomycin, and L-glutamine.

\section{Depletion of PGRMC1 transcripts in HepG2 cells}

HepG2 cells were transfected with SMARTpool ON-TARGET plus (GE Dharmacon) siRNAs directed against human PGRMC1 (30 nM) using Lipofectamine RNAi-MAX (Invitrogen). As a negative control, cells were transfected with ON-TARGET plus (GE Dharmacon) nontargeting pool siRNAs (30 nM). Twenty-four hours after transfection, the media were replaced with serum-free EMEM. Cells were harvested 52 hours after the addition of serum-free media. Cells were exposed to progesterone $(30 \mu \mathrm{M})$ or mifepristone $(30 \mu \mathrm{M}) 12$ hours prior to sample collection, epitiostanol (30 M) 24 hours prior to sample collection, BMP6 $(20 \mathrm{ng} / \mathrm{ml}$; R\&D Systems) 3 hours prior to sample collection, or IL-6 (100 ng/ml; R\&D Systems) 3 hours prior to sample collection.

\section{Antibody blockade of PGRMC1 in HepG2 cells}

HepG2 cells were incubated in serum-free EMEM, and, 18 hours later, cells were treated with polyclonal antibody directed against the extracellular domain of PGRMC1 (H-46, sc-98680, Santa Cruz) at a final concentration of $10 \mu \mathrm{g} / \mathrm{ml}$ for 4 hours or isotype-matched, rabbit IgG (sc-2027, Santa Cruz) at the same final concentration. Cells were then exposed to progesterone $(30 \mu \mathrm{M})$ or mifepristone $(30 \mu \mathrm{M}) 8$ hours prior to sample collection, to BMP6 $(20 \mathrm{ng} / \mathrm{ml})$ 3 hours prior to sample collection, or to IL-6 (100 ng/ml) 3 hours prior to sample collection.

\section{Quantitative real-time PCR analysis}

Zebrafish. Zebrafish total RNA was extracted from embryos using the RNeasy Mini Kit (Qiagen). First-strand cDNA was synthesized by reverse transcription from total RNA using the QuantiTech Reverse Transcription Kit (Qiagen). For qPCR analysis, the ABI Fast SYBR Green Master Mix and 7500 fast PCR machine were used for the amplification and quantification of transcripts. Relative expression of target transcripts was normalized to gapdh mRNA levels using the relative $\mathrm{C}_{\mathrm{T}}$ method. All results were compared with those in fish treated with vehicle control. The primer sequences used for qPCR are as follows: hepcidin-F, CTGGCTGCTGTC- 
GTCAT; hepcidin-R, TGGTTCTCCTGCAGTTCTTCAC; GAPDH-F, AGGCTTCTCACAAACGAGGA; GAPDH-R, GATGGCCACAATCTCCACTT; ferroportin-F, TGCTCTTACTGGGCGCTATT; ferroportin-R, ATGTTGTCAGCAACCATCCA; id1-F, GCATCTCTGTGGAGAACGG; id1-R, CGATGCTTCGATGATCTGTT; liv1-F, GTCATCATGGGAGACGGACT; liv1-R, GAAGCTCATGGCAGAACACA.

HepG2 cells and mouse liver. Total RNA was extracted from HepG2 cells and mouse liver samples using TRIzol (Invitrogen). For reverse RNA transcription, $1 \mu \mathrm{g}$ of RNA samples was reverse transcribed to cDNA using random hexamer primers and MMLV-RT (Applied Biosystems) in a $20-\mu \mathrm{l}$ reaction. cDNA samples were then diluted at a ratio of 1:4, and $6 \mu$ of this cDNA pool was used for quantitative PCR in a 20- $\mu \mathrm{l}$ reaction using TaqMan Fast Advanced Master Mix (Invitrogen) and specific TaqMan probes (HAMP, Hs00221783 m1; Hamp, Mm04231240_s1; 18s, Hs03928985_g1; PGRMC1, Hs00998344_m1; and TBP, Mm00446971_m1; Thermo Fisher Scientific). A Mastercycler ep Realplex (Eppendorf) was used for real-time amplification and quantification of transcripts. Relative expression of target transcripts was normalized to levels of 18 s ribosomal RNA or Tbp mRNA using the relative $\mathrm{C}_{\mathrm{T}}$ method. TaqMan gene expression assays were used to quantify mRNA levels of $18 \mathrm{~s}$ ribosomal RNA and mRNAs encoding hepcidin, PGRMC1, or TBP. All results were compared with those of cells or mice treated with vehicle control.

\section{Measurement of phosphorylated BMP-responsive SMAD levels and phosphorylated STAT3}

HepG2 cells were incubated in serum-free media for 18 hours and then exposed to progesterone $(30 \mu \mathrm{M})$ or mifepristone $(30 \mu \mathrm{M})$ for various intervals or BMP6 $(20 \mathrm{ng} / \mathrm{ml})$ or IL-6 $(100 \mathrm{ng} / \mathrm{ml})$ for 3 hours. Cells were harvested in radioimmunoprecipitation assay buffer supplemented with protease and phosphatase inhibitors (Sigma-Aldrich). Western blots were performed on HepG2 cell lysates using antibodies directed against total SMAD1 (catalog LS-C75853; LifeSpan BioSciences Inc.), phosphorylated SMAD1/5 (catalog 9516; Cell Signaling Technology), total STAT3 (catalog 49045; Cell Signaling Technology), and STAT3 phosphorylated at tyrosine 705 (catalog 9145L; Cell Signaling Technology) as described previously (64). These antibodies have been used by other investigators to evaluate levels of total SMAD1, phosphorylated SMAD1/5, total STAT3, and STAT3 phosphorylated at tyrosine $705(22,64)$.

\section{BMP-responsive element and hepcidin promoter luciferase reporter assays}

HepG2 cells grown to $60 \%$ confluence in 6-well plates were transiently transfected with $I d 1$ promoter luciferase reporter construct (BRE-Luc; $0.25 \mu \mathrm{g}$ per well) (20) or hepcidin promoter luciferase reporter (Hep-Luc; $0.25 \mu$ g per well) (22) using Xtreme Gene transfection reagent (Roche Life Science). A plasmid encoding Renilla luciferase (pRL-TK; Promega Life Sciences) was used to control for transfection efficiency. Twenty-four hours after transfection, HepG 2 cells were incubated in serum-free EMEM for 18 hours. Cells were then exposed to BMP6 (20 ng/ml), IL-6 (100 ng/ml), progesterone $(30 \mu \mathrm{M})$, or mifepristone $(30 \mu \mathrm{M})$ for 24 hours. Cell extracts were harvested, and the Dual Luciferase Assay Kit (Promega) was used to quantify relative promoter activity by measuring the ratio of firefly to Renilla luciferase activity. All results were normalized to those in cells treated with vehicle alone.

\section{Mice}

Ten-week-old female mice were fed an iron-deficient diet (2-5 ppm iron, Teklad diet TD.80396, Harlan Laboratories) for 14 days prior to injection with vehicle or mifepristone. Mifepristone was diluted in a solution containing diethylene glycol monoethyl ether (SigmaAldrich), Cremophor EL (Fluka Biochemka), and phosphate-buffered saline in a 1:1:3 ratio. Mice received an intraperitoneal injection of either mifepristone $(100 \mathrm{mg} / \mathrm{kg})$ or vehicle alone, and liver tissue was harvested for RNA extraction 10 hours later.

\section{Serum hepcidin measurements}

Serum samples were prepared from peripheral blood and stored at $-80^{\circ} \mathrm{C}$ until assayed. Levels of hepcidin- 25 in human serum samples were measured using a competitive ELISA from either DRG International Inc. (EIA-5258) or Peninsula Laboratories International (S-1337.0001, formerly distributed by Bachem) according to the manufacturers' protocol. All samples were tested in duplicate, and the level of hepcidin was determined by comparison to dilutions of recombinant protein standards. Intra-assay variability for the ELISA from DRG International Inc. was 2.6\%, and the inter-assay variability for the ELISA from DRG International Inc. was $4.1 \%$.

\section{Statistics}

All values are expressed as mean \pm SEM. All graphs were generated using GraphPad Prism Software. Data were analyzed using the 2-tailed Student's $t$ test, 1-way ANOVA, or 2-way ANOVA. $P$ values of less than 0.05 were considered significant.

\section{Study approval}

Animals. All animal experiments were approved by the Subcommittee on Research Animal Care of the Massachusetts General Hospital.

Human serum samples. The Institutional Review Boards at the Massachusetts General Hospital (protocol 2015P001205) and New York University Langone Medical Center (protocol i15-0078) approved the studies performed on discarded anonymized human serum samples.

\section{Author contributions}

$\mathrm{XL}$ and DKR designed and performed the experiments, analyzed the data, and wrote the manuscript. CM, RM, EA, LAH, and GS designed and performed the experiments, acquired the data, and contributed to data analysis. YK, DM, and DK provided reagents, designed the experiments, acquired the data, and contributed to data analysis. KDB designed the experiments and interpreted the data. DBB and RTP designed the experiments, interpreted the data, and wrote the manuscript.

\section{Acknowledgments}

Support was provided by NIH grant R01DK082971 (to K.D. Bloch, D.B. Bloch, and R.T. Peterson), National Heart, Lung, and Blood Institute (NHLBI) training grant T32HL007208 (to D.K. Rhee), the John S. LaDue Memorial Fellowship from Harvard Medical School (to D.K. Rhee), NHLBI grant K08HL111210 (to R. Malhotra), the Charles and Ann Sanders Massachusetts General Hospital Scholar Award (to R.T. Peterson), and the Leducq Foundation (to C. Mayeur, L.A. Hurst, G. Shelton, D.B. Bloch, and K.D. Bloch). 
Address correspondence to: Randall T. Peterson, Massachusetts General Hospital, Cardiovascular Research Center, 149 13th Street, 4.101A, Charlestown, Massachusetts 02129, USA. Phone: 617.724.9569; E-mail: peterson@cvrc.mgh.harvard.edu. Or to:
Donald B. Bloch, Massachusetts General Hospital, Anesthesia Center for Critical Care Research, Thier Building 505, Boston, Massachusetts 02114, USA. Phone: 617.726.3780; E-mail: bloch@helix. mgh.harvard.edu.
1. Zhao N, Zhang AS, Enns CA. Iron regulation by hepcidin. J Clin Invest. 2013;123(6):2337-2343.

2. Evstatiev R, Gasche C. Iron sensing and signalling. Gut. 2012;61(6):933-952.

3. Nemeth E, et al. Hepcidin regulates cellular iron efflux by binding to ferroportin and inducing its internalization. Science. 2004;306(5704):2090-2093.

4. Kautz L, Jung G, Valore EV, Rivella S, Nemeth $\mathrm{E}, \mathrm{Ganz}$ T. Identification of erythroferrone as an erythroid regulator of iron metabolism. Nat Genet. 2014;46(7):678-684.

5. Andriopoulos B Jr, et al. BMP6 is a key endogenous regulator of hepcidin expression and iron metabolism. Nat Genet. 2009;41(4):482-487.

6 . Babitt JL, et al. Bone morphogenetic protein signaling by hemojuvelin regulates hepcidin expression. Nat Genet. 2006;38(5):531-539.

7. Meynard D, Kautz L, Darnaud V, Canonne-Hergaux F, Coppin H, Roth MP. Lack of the bone morphogenetic protein BMP6 induces massive iron overload. Nat Genet. 2009;41(4):478-481.

8. Nemeth E, et al. IL-6 mediates hypoferremia of inflammation by inducing the synthesis of the iron regulatory hormone hepcidin. J Clin Invest. 2004;113(9):1271-1276.

9. Verga Falzacappa MV, Vujic Spasic M, Kessler R, Stolte J, Hentze MW, Muckenthaler MU. STAT3 mediates hepatic hepcidin expression and its inflammatory stimulation. Blood. 2007;109(1):353-358.

10. Wrighting DM, Andrews NC. Interleukin-6 induces hepcidin expression through STAT3. Blood. 2006;108(9):3204-3209.

11. Pasricha SR, Frazer DM, Bowden DK, Anderson GJ. Transfusion suppresses erythropoiesis and increases hepcidin in adult patients with $\beta$-thalassemia major: a longitudinal study. Blood. 2013;122(1):124-133.

12. Gardenghi S, et al. Ineffective erythropoiesis in beta-thalassemia is characterized by increased iron absorption mediated by down-regulation of hepcidin and up-regulation of ferroportin. Blood. 2007;109(11):5027-5035.

13. Adams PC, Barton JC. How I treat hemochromatosis. Blood. 2010;116(3):317-325.

14. Borgna-Pignatti C, et al. Survival and complications in patients with thalassemia major treated with transfusion and deferoxamine. Haematologica. 2004;89(10):1187-1193.

15. Neufeld EJ. Update on iron chelators in thalassemia. Hematology Am Soc Hematol Educ Program. 2010;2010:451-455.

16. Preza GC, et al. Minihepcidins are rationally designed small peptides that mimic hepcidin activity in mice and may be useful for the treatment of iron overload. JClin Invest. 2011;121(12):4880-4888.

17. Nai A, et al. Deletion of TMPRSS6 attenuates the phenotype in a mouse model of beta-thalassemia. Blood. 2012;119(21):5021-5029.
18. Ramos E, et al. Minihepcidins prevent iron overload in a hepcidin-deficient mouse model of severe hemochromatosis. Blood. 2012;120(18):3829-3836.

19. Schmidt PJ, et al. An RNAi therapeutic targeting Tmprss 6 decreases iron overload in $\mathrm{Hfe}^{(-/)}$ mice and ameliorates anemia and iron overload in murine $\beta$-thalassemia intermedia. Blood. 2013;121(7):1200-1208

20. Korchynskyi O, ten Dijke P. Identification and functional characterization of distinct critically important bone morphogenetic protein-specific response elements in the Id1 promoter. J Biol Chem. 2002;277(7):4883-4891.

21. Casanovas G, Mleczko-Sanecka K, Altamura S, Hentze MW, Muckenthaler MU. Bone morphogenetic protein (BMP)-responsive elements located in the proximal and distal hepcidin promoter are critical for its response to HJV/BMP/ SMAD. J Mol Med (Berl). 2009;87(5):471-480.

22. Yu PB, et al. Dorsomorphin inhibits BMP signals required for embryogenesis and iron metabolism. Nat Chem Biol. 2008;4(1):33-41.

23. Zhang K, Chow PK. The effect of megestrol acetate on growth of HepG2 cells in vitro and in vivo. Clin Cancer Res. 2004;10(15):5226-5232.

24. Cahill MA. Progesterone receptor membrane component 1: an integrative review. J Steroid Biochem Mol Biol. 2007;105(1-5):16-36.

25. Rohe HJ, Ahmed IS, Twist KE, Craven RJ. PGRMC1 (progesterone receptor membrane component 1): a targetable protein with multiple functions in steroid signaling, P450 activation and drug binding. Pharmacol Ther. 2009;121(1):14-19.

26. Engmann L, Losel R, Wehling M, Peluso JJ. Progesterone regulation of human granulosa/ luteal cell viability by an RU486-independent mechanism. J Clin Endocrinol Metab. 2006;91(12):4962-4968.

27. Zhao $\mathrm{X}$, et al. Interruption of cenph causes mitotic failure and embryonic death, and its haploinsufficiency suppresses cancer in zebrafish. J Biol Chem. 2010;285(36):27924-27934.

28. Swiatek-De Lange M, et al. Membrane-initiated effects of progesterone on calcium dependent signaling and activation of VEGF gene expression in retinal glial cells. Glia. 2007;55(10):1061-1073.

29. Zhang M, et al. Progesterone receptor membrane component 1 is a functional part of the glucagon-like peptide-1 (GLP-1) receptor complex in pancreatic beta cells. Mol Cell Proteomics. 2014;13(11):3049-3062.

30. Hanke JH, et al. Discovery of a novel, potent, and Src family-selective tyrosine kinase inhibitor. Study of Lck- and FynT-dependent T cell activation. J Biol Chem. 1996;271(2):695-701.

31. Hou XY, Liu Y, Zhang GY. PP2, a potent inhibitor of Src family kinases, protects against hippocampal CA1 pyramidal cell death after transient global brain ischemia. Neurosci Lett.
2007;420(3):235-239.

32. Vandendriessche $\mathrm{B}$, et al. The soluble guanylate cyclase activator BAY 58-2667 protects against morbidity and mortality in endotoxic shock by recoupling organ systems. PLoS One. 2013;8(8):e72155.

33. van der Linden M, Buckingham K, Farquhar C, Kremer JA, Metwally M. Luteal phase support for assisted reproduction cycles. Cochrane Database Syst Rev. 2011;(10):CD009154.

34. Beggs LA, et al. Testosterone alters iron metabolism and stimulates red blood cell production independently of dihydrotestosterone. Am JPhysiol Endocrinol Metab. 2014;307(5):E456-E461.

35. Casals-Pascual C, et al. Hepcidin demonstrates a biphasic association with anemia in acute Plasmodium falciparum malaria. Haematologica. 2012;97(11):1695-1698.

36. Dao MC, Sen S, Iyer C, Klebenov D, Meydani SN. Obesity during pregnancy and fetal iron status: is Hepcidin the link? J Perinatol. 2013;33(3):177-181.

37. Talbot NP, et al. Regulation of hepcidin expression at high altitude. Blood. 2012;119(3):857-860.

38. Steinbicker AU, et al. Inhibition of bone morphogenetic protein signaling attenuates anemia associated with inflammation. Blood. 2011;117(18):4915-4923.

39. Sun CC, Vaja V, Babitt JL, Lin HY. Targeting the hepcidin-ferroportin axis to develop new treatment strategies for anemia of chronic disease and anemia of inflammation. Am J Hematol. 2012;87(4):392-400.

40. Babitt JL, Huang FW, Xia Y, Sidis Y, Andrews NC, Lin HY. Modulation of bone morphogenetic protein signaling in vivo regulates systemic iron balance. J Clin Invest. 2007;117(7):1933-1939.

41. Meyer C, Schmid R, Scriba PC, Wehling M. Purification and partial sequencing of high-affinity progesterone-binding site(s) from porcine liver membranes. Eur J Biochem. 1996;239(3):726-731.

42. Min L, et al. Molecular identification of adrenal inner zone antigen as a heme-binding protein. FEBS J. 2005;272(22):5832-5843.

43. Peluso JJ, Pru JK. Non-canonical progesterone signaling in granulosa cell function. Reproduction. 2014;147(5):R169-R178.

44. Peluso JJ, Liu X, Saunders MM, Claffey KP, Phoenix K. Regulation of ovarian cancer cell viability and sensitivity to cisplatin by progesterone receptor membrane component-1. J Clin Endocrinol Metab. 2008;93(5):1592-1599.

45. Peluso JJ, Pappalardo A, Losel R, Wehling M. Progesterone membrane receptor component 1 expression in the immature rat ovary and its role in mediating progesterone's antiapoptotic action. Endocrinology. 2006;147(6):3133-3140.

46. Hughes AL, et al. Dap1/PGRMC1 binds and regulates cytochrome $\mathrm{P} 450$ enzymes. Cell Metab. 2007;5(2):143-149.

47. Mallory JC, et al. Dap1p, a heme-binding protein that regulates the cytochrome $\mathrm{P} 450$ protein 
Erg11p/Cyp51p in Saccharomyces cerevisiae. Mol Cell Biol. 2005;25(5):1669-1679.

48. Craven RJ, Mallory JC, Hand RA. Regulation of iron homeostasis mediated by the heme-binding protein Dap1 (damage resistance protein 1) via the P450 protein Erg11/Cyp51. J Biol Chem. 2007;282(50):36543-36551.

49. Peluso JJ, DeCerbo J, Lodde V. Evidence for a genomic mechanism of action for progesterone receptor membrane component-1. Steroids. 2012;77(10):1007-1012.

50. Meynard D, et al. Regulation of TMPRSS6 by BMP6 and iron in human cells and mice. Blood. 2011;118(3):747-756.

51. Hou Y, et al. Estrogen regulates iron homeostasis through governing hepatic hepcidin expression via an estrogen response element. Gene. 2012;511(2):398-403.

52. Ikeda Y, et al. Estrogen regulates hepcidin expression via GPR30-BMP6-dependent signaling in hepatocytes. PLoS One. 2012;7(7):e40465.

53. Yang Q, Jian J, Katz S, Abramson SB, Huang X.
17beta-Estradiol inhibits iron hormone hepcidin through an estrogen responsive element half-site. Endocrinology. 2012;153(7):3170-3178.

54. Guo W, et al. Testosterone administration inhibits hepcidin transcription and is associated with increased iron incorporation into red blood cells. Aging Cell. 2013;12(2):280-291.

55. Latour C, et al. Testosterone perturbs systemic iron balance through activation of epidermal growth factor receptor signaling in the liver and repression of hepcidin. Hepatology. 2014;59(2):683-694.

56. Kong WN, et al. Sex differences in iron status and hepcidin expression in rats. Biol Trace Elem Res. 2014;160(2):258-267.

57. Galesloot TE, et al. Serum hepcidin: reference ranges and biochemical correlates in the general population. Blood.2011;117(25):e218-e225.

58. Ganz T, Olbina G, Girelli D, Nemeth E, Westerman M. Immunoassay for human serum hepcidin. Blood. 2008;112(10):4292-4297.

59. Koenig MD, Tussing-Humphreys L, Day J,
Cadwell B, Nemeth E. Hepcidin and iron homeostasis during pregnancy. Nutrients. 2014;6(8):3062-3083.

60. Apgar BS, Greenberg G. Using progestins in clinical practice. Am Fam Physician. 2000;62(8):1839-1846.

61. Fleseriu M, et al. Mifepristone, a glucocorticoid receptor antagonist, produces clinical and metabolic benefits in patients with Cushing's syndrome. JClin Endocrinol Metab. 2012;97(6):2039-2049.

62. Li X, Jia S, Wang S, Wang Y, Meng A. Mta3-NuRD complex is a master regulator for initiation of primitive hematopoiesis in vertebrate embryos. Blood. 2009;114(27):5464-5472.

63. Fraenkel PG, et al. Transferrin-a modulates hepcidin expression in zebrafish embryos. Blood. 2009;113(12):2843-2850.

64. Mayeur C, et al. The type I BMP receptor Alk3 is required for the induction of hepatic hepcidin gene expression by interleukin-6. Blood. 2014;123(14):2261-2268. 